Research Paper

\title{
Cytokine-Induced Killer Cells Modulates Resistance to Cisplatin in the A549/DDP Cell Line
} \author{
$\mathrm{Yu}^{1,3,4,5,6 \text {, Shui Cao }}{ }^{2,3,4,5,6}$, Hui Li1,3,4,5,6凶 , Xiubao Ren 1, 2, 3,4, 5,6凶 \\ 1. Department of Immunology, Tianjin Medical University Cancer Institute and Hospital, Tianjin, China; \\ 2. Department of Biotherapy, Tianjin Medical University Cancer Institute and Hospital, Tianjin, China; \\ 3. National Clinical Research Center for Cancer, Tianjin, China; \\ 4. Key Laboratory of Cancer Immunology and Biotherapy, Tianjin, China; \\ 5. Key Laboratory of Cancer Prevention and Therapy, Tianjin, China; \\ 6. Tianjin's Clinical Research Center for Cancer, Tianjin, China. \\ * These authors contributed equally to this article.
}

Lili Yang1, 3, 4, 5, 6*, Chunjuan Du1,3,4,5,6*, Lei Wu1,3,4,5,6, Jinpu Yu1,3,4,5,6, Xiumei An1,3,4,5, 6, Wenwen

$\triangle$ Corresponding authors: Xiubao Ren and Hui Li; Xiubao Ren, MD. Ph.D., Huanhuxi Road, Tiyuanbei, Hexi District, Tianjin, P. R. China, 300060 E-mail address: rwziyi@yahoo.com;Phone: 086-022-23340123;Fax:086-022-23537796 Hui Li, MD., Ph.D.,_Huanhuxi Road, Tiyuanbei, Hexi District, Tianjin, P. R. China, 300060 E-mail address: lihui@tjmuch.com; Phone: 086-022-23340123;Fax: 086-022-23537796

(C) Ivyspring International Publisher. This is an open access article distributed under the terms of the Creative Commons Attribution (CC BY-NC) license (https://creativecommons.org/licenses/by-nc/4.0/). See http://ivyspring.com/terms for full terms and conditions.

Received: 2017.01.31; Accepted: 2017.08.30; Published: 2017.09.16

\begin{abstract}
Background Cytokine-induced killer (CIK) cells can potentially enhance the tumor-killing activity of chemotherapy. Objective This study aimed to evaluate the effects of CIK cells on cisplatin (DDP) resistance in the human lung adenocarcinoma cell line A549/DDP. Methods The detect resistance index, drug resistance related-genes and cytokine secretion of A549/DDP co-cultured with CIK cells were assayed in vitro. Results After A549/DDP co-culture with CIK cells, the DDP resistance of A549/DDP significantly decreased in a time-dependent manner. The DDP resistance of A549/DDP co-cultured with CIK cells for $20 \mathrm{~h}$ decreased 4.93-fold compared with that of A549/DDP cells cultured alone $(P<0.05)$. The mRNA and protein expression levels of the glutathione-S-transferase (GST) $-\pi$ gene in A549/DDP significantly decreased after co-culture with CIK cells $(P<0.05)$. The secretion of interferon (IFN)- $\gamma$ significantly increased along with the co-culture time of A549/DDP with CIK cells. The expression of GST- $\pi$ was restored by adding the neutralizing IFN- $\gamma$. Conclusion CIK cells can reverse the drug resistance of A549/DDP in a time-dependent manner by reducing GST- $\pi$ expression to increase the accumulation of DDP. The effect of CIK cells on re-sensitizing lung cancer cells to the chemotherapy drug was partially dependent on the secretion of IFN- $\gamma$.
\end{abstract}

Key words: cytokine-induced killer cells; chemotherapy resistance; lung cancer; GST- $\pi$; IFN- $\gamma$.

\section{Introduction}

Chemotherapy is a commonly used adjuvant therapy for malignant tumors. Despite the progress in improving chemotherapy outcome, individual differences in drug response have led to diverse efficacy in cancer patients, and many patients suffer from suboptimal response or resistance to chemotherapy drugs. Chemotherapy resistance has become a main obstacle undermining chemotherapeutic effects.
Lung cancer is a major cause of cancer deaths with a 5-year survival rate of less than $15 \%$ [1]. Non-small cell lung cancer (NSCLC) accounts for approximately $85 \%-90 \%$ of all cases of lung cancer [2]. For more than two decades, the most effective systemic chemotherapy for NSCLC was cisplatin (DDP) or other platinum based combinations, which remain the standard first-line chemotherapy for 
advanced NSCLC [3]. DDP resistance is a major obstacle for its clinical effectiveness in NSCLC.

Cytokine-induced killer (CIK) cells are a unique population of cytotoxic $\mathrm{T}$ lymphocytes with the characteristic CD3+CD56+ phenotype [4]. CIK cells can proliferate abundantly in vitro and kill tumor cells directly. CIK cells are considered a new generation of tumor-killing cells as an adoptive immunotherapy [5]. Recently, CIKs have been reported for their potential function to enhance the tumor-killing effect of chemotherapy and improve the treatment outcome when combined with chemotherapy, but the mechanism of this synergism is unclear [6-8]. We hypothesize that CIK cells enhance the efficacy of chemotherapy by reversing the drug resistant phenotype of tumor cells. The present study determined whether CIK cells can reverse drug resistance and further explore its mechanism to seek a new and effective treatment for drug resistant lung cancer.

\section{Materials and Methods}

\section{Cell Culture}

The human lung adenocarcinoma cell line A549 and the DDP-resistant cell line A549/DDP were purchased from the Tumor Cell Bank of the Chinese Academy of Medical Science and maintained in RPMI 1640 (TAKARA, Japan) containing 10\% heat-inactivated fetal bovine serum (GIBCO, USA) in a humidified incubator with $5 \% \quad \mathrm{CO}_{2}$ at $37^{\circ} \mathrm{C}$. A549/DDP cells were cultured in the presence of

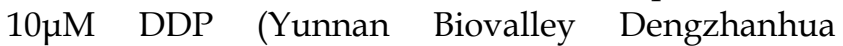
Pharmaceutical Co. Ltd, China), which was withdrawn for two generations before the experiments were performed. These cell lines grew in monolayers and were passaged when the cultures were $70-80 \%$ confluent.

\section{CIK cells preparation}

Auto-CIK cells were generated as previously determined $[9,10]$. Peripheral blood mononuclear cells (PBMCs) were collected from NSCLC patients using Cobe Spectra Apheresis System (CaridianBCT, USA). CIK cells were developed as follows: The cells were cultured in X-VIVO 20 serum-free medium (Cambrex, USA), consisting of $100 \mathrm{U} / \mathrm{mL}$ recombinant human IL-1a, $50 \mathrm{ng} / \mathrm{mL}$ anti-CD3 antibody (e-Bioscience, USA), and 1,000 U/ mL recombinant human IFN- $\gamma$ (PEPROTECH, USA) at 37 ${ }^{\circ} \mathrm{C}$ in a humidified atmosphere with $5 \% \mathrm{CO}_{2}$ for $24 \mathrm{~h}$ before $300 \mathrm{U} / \mathrm{mL}$ recombinant human IL-2 (PEPROTECH, USA) was added to the medium. IL-2 and IFN- $\gamma$ containing medium was added to the culture system every 5 days. At day 14, the cells were harvested and analyzed for phenotype and cytotoxicity. The phenotypes of CIK were analyzed by flow cytometry (BD Aria, USA) with antibodies against CD3- PerCP5.5, CD4-FITC, CD56-PE, CD25-APC (BD Bioscience, USA).

\section{Co-culture assay}

A549/DDP was co-cultured with CIK cells using $0.4 \mu \mathrm{m}$ pore size transwells (Corning, USA). A549/DDP $\left(2.5 \times 10^{5} / \mathrm{mL}\right)$ was seeded in the lower chamber of 24-well plates, whereas CIK cells were added to the upper chamber at the ratio of 20:1 (CIK cells: A549/DDP). Cell-free medium was added to the upper chamber in the control groups. Co-culture time was controlled at $6,12,16$ and $20 \mathrm{~h}$.

\section{Drug sensitivity assay}

A549 and A549/DDP grown in the logarithmic phase and A549/DDP co-cultured with CIK cells were harvested and dissociated using $0.25 \%$ trypsin-EDTA and then seeded at 5000 cells per well in 96-well plates. Triplicate wells were used for each determination. An overnight preincubation time was allowed after cell seeding prior to addition of DDP. DDP was added to the cells at seven increasing concentrations. Blank wells only contained cell-free medium. After $48 \mathrm{~h}$ incubation with DDP, $20 \mu \mathrm{L}$ of MTT (5 mg/mL, Sigma, St. Louis, MO, USA) was added to each well and the plates were incubated at $37^{\circ} \mathrm{C}$ for $4 \mathrm{~h}$. The formazan crystals were dissolved in $150 \mu \mathrm{L}$ of DMSO (Sigma) and the absorbance (OD) was measured at $570 \mathrm{~nm}$ using a spectrophotometer (Synergy HT Multi-Mode Microplate Reader, BioTek, USA). Blank wells for absorbance readings contained cell-free medium. All experiments were performed at least three times. The growth inhibitory rate was calculated by using the following formula: Growth inhibitory rate $(\mathrm{IR})=($ average OD value in the control group - average OD value in the treatment group)/average OD value in the control group $x$ $100 \%$. Then the linear regression between $\log 10$ (drug concentrations) and cell growth inhibition rates was used to determine the half inhibition concentration (IC50). Resistance index (RI) $=$ IC50 (A549/DDP)/ IC50 (A549). Reversal index $=$ IC50 (A549/DDP)/IC50 (A549/DDP co-cultured with CIK cells).

\section{mRNA and protein expression of drug resistance-associated genes}

The expression of glutathione-S-transferase (GST- $\pi)$, multidrug resistance 1 (MDR1), human copper transporter 1 ( $h C T R 1)$, excision repair cross complementing 1 (ERCC1), and the copper transporters $A T P 7 A$ and $A T P 7 B$ were detected using RT-PCR. Total RNA was extracted from A549 and A549/DDP cells using TRIzol reagent (Sigma, USA). First-strand cDNA was synthesized by reverse 
transcription. The sequences of primers used and the product lengths are shown in Table $\mathbf{1}$. Changes in the expression of resistance-related genes in A549, A549/DDP and A549/DDP cells co-cultured with CIK cells were examined using real-time RT-PCR. SYBR® Premix Ex Taq ${ }^{\mathrm{TM}}$ (TAKARA, Japan) was used for real-time PCR. The CT value of each gene was generated automatically by ABI PRISM 7500. The gene copy number was calculated by $2-\triangle \mathrm{CT}(\triangle \mathrm{CT}=\mathrm{CT}$ target gene- $\mathrm{CT}$ internal reference gene). The protein levels of GST $-\pi$ and hCTR1 were detected from A549, A549/DDP and A549/DDP cells co-cultured with CIK cells accordance with the standard protocol.

\section{Enzyme-Linked Immunosorbent Assay (ELISA) for cytokines secretion}

The secretion levels of IFN- $\gamma$, IL-2, and TNF- $\alpha$ in the co-culture supernatants of A549/DDP and CIKs were detected using ELISA in accordance with the manufacturer's instructions (Shenzhen Dakota for the Biotechnology Co.Ltd, China).

\section{Antibody neutralization test}

Anti-human IFN- $\gamma$ antibody, anti-human TNF- $\alpha$ antibody, or anti-human IL-2 antibody $(1 \mu \mathrm{g} / \mathrm{mL}$; all purchased from Biolegend, USA) was added to the co-culture system of A549/DDP and CIK cells to neutralize these cytokines secreted by CIK cells. A549, A549/DDP cultured alone and A549/DDP co-cultured with CIK cells without the neutralizing antibody treatment were set as the control groups. The DDP resistance of these cells and the expression levels of GST- $\pi$ were detected.

\section{Analysis of drug accumulation by high-performance liquid chromatography (HPLC)}

One day after the cells were seeded, DDP (10 $\mu \mathrm{g} / \mathrm{mL}$ ) was added to the flasks. The cells were harvested after $6 \mathrm{~h}$. After washing by three times with RPMI-1640, cell pellets were collected and resuspended in $100 \mu \mathrm{L}$ of saline per $10^{7}$ cells, followed by freeze/thaw for 5 times to lyse the cells. After centrifugation at $12,000 \mathrm{rpm}$ for $30 \mathrm{~m}$, the supernatant was collected. Using $100 \mu \mathrm{L}$ of the supernatant as the test solution, $100 \mu \mathrm{L}$ of methanol was added, vortexed, and then centrifuged at 13,000 rpm for 15 min. The precipitate was discarded, and $10 \mu \mathrm{L}$ of $5 \%$ DDTC $(0.1 \% \mathrm{NaOH}$ preparation) was added to the supernatant and incubated for $20 \mathrm{~min}$ at $37^{\circ} \mathrm{C}$. Then, $100 \mu \mathrm{L}$ of chloroform was added, and the mixture was vortexed for $2 \mathrm{~min}$ and centrifuged at 10,000 rpm for $5 \mathrm{~min}$. The chloroform layer was collected to detect DDP accumulation. DDP solutions at different concentrations of $0.2,0.5,1,1.5,10$ and $20 \mu \mathrm{g} / \mathrm{mL}$ were prepared for the standard curve for calibration. DDP accumulation in different cells was quantified by HPLC using Kromasil C- 18 columns $(25 \mathrm{~cm} \times 4.6 \mathrm{~mm}$, $5 \mu \mathrm{m})$ (Alltech, Deerfield, IL) with the mobile phase consisting of a ternary mixture of methanol, acetonitrile and water $(40: 30: 30)$ at a flow rate of 1.0 $\mathrm{mL} / \mathrm{min}$ and spectrophotometric detection at $254 \mathrm{~nm}$. The HPLC (Alliance 2695, Waters) was equipped with a UV detector (2489 UV/Visible Detector, Waters), and a Chromatography Empower 2.0 software.

\section{Statistical analysis}

Results were presented as mean $\pm \mathrm{SD}$. We used one-way ANOVA for comparisons between different cell lines under different conditions, and correlation analysis for the association between resistance reversal and cytokines secreted by CIK cells. $P<0.05$ was considered statistically significant.

\section{Results}

\section{Reversal of drug resistance of A549/DDP after co-culture with CIK cells}

The response of A549 and A549/DDP to DDP was measured by MTT. The half inhibitory concentrations (IC50) of A549 and A549/DDP were $5.0 \pm 1.25$ and $72.6 \pm 4.65 \mu \mathrm{mol} / \mathrm{L}$, respectively. The RI of A549/DDP was 14.5, indicating moderate DDP resistance (Table 2). After co-culture with CIK cells, the DDP resistance of A549/DDP significantly decreased in a time-dependent manner. The DDP resistance of the A549/DDP co-cultured with CIK cells for 20h decreased 4.93-fold compared with that of the A549/DDP cells cultured alone $(P<0.05)$ (Table 2).

Table 1. Primers used in the study

\begin{tabular}{llll}
\hline Gene & Forward/Reverse primers $\left(5^{\prime}-3^{\prime}\right)$ & Production length $(\mathrm{bp})$ & Annealing temperature $\left({ }^{\circ} \mathrm{C}\right)$ \\
\hline GST- $\pi$ & CAGTCCAATACCATCCTGCGT CACGTCATCCTTGCCCGCCTCATAG & 162 & 60 \\
$h C t r 1$ & 'GCTGGAAGAAGGCAGTGGTAGGCACAAAGAGGAGCAAGAAGG & 150 & 60 \\
ERCC1 & AGCAGAAACCAGCGGACCTCCTCTTGATGCGGCGATGAGC & 159 & 60 \\
MDR 1 & GGAAGCCAAATGCCTATGACTCGATGAGCTATCACAATGGT & 181 & 62 \\
ATP7B & TGCAAAGTCAGCAACGTGGACATCTCGTGGTCTGTCATAGCG & 213 & 58 \\
ATP7A & ACTGCAAGGTGTTCAGCGAATGCTGTGACCCTTCTGAGGATTT & 218 & 58 \\
$\beta$-actin & TGGCACCCAGCACAATGAACTAAGTCATAGTCCGCCTAGAAGCA' & 186 & 60 \\
\hline
\end{tabular}


Table 2. DDP resistance of different cell groups.

\begin{tabular}{llll}
\hline Cells & IC50 $(\mu \mathrm{mol} / \mathrm{L})$ & $\begin{array}{l}\text { Resistance } \\
\text { index }\end{array}$ & $\begin{array}{l}\text { Reversal } \\
\text { index }\end{array}$ \\
\hline A549 & $5.0 \pm 1.25$ & & \\
A549/DDP & $72.6 \pm 4.65$ & 14.5 & \\
A549/DDP co-cultured for 6h & $25.88 \pm 4.35$ & 5.2 & 2.79 \\
A549/DDP co-cultured for 12h & $18.33 \pm 2.98$ & 3.67 & 3.95 \\
A549/DDP co-cultured for 16h & $15.65 \pm 3.74$ & 3.13 & 4.63 \\
A549/DDP co-cultured for 20h & $14.70 \pm 2.12^{*}$ & 2.94 & 4.93 \\
\hline
\end{tabular}

Results were presented as mean \pm SD. We used one-way ANOVA for comparisons between different cell lines under different conditions. ${ }^{*} P<0.05$ compared with A549/DDP alone.

\section{Analysis of drug resistance related-genes of A549/DDP after co-culture with CIK cells}

To identify drug resistance associated-genes, the expression levels of hCTR1, ATP7A, ATP7B, MDR1, GST- $\pi$ and ERCC1 in A549 and A549/DDP were analyzed by quantitative real-time (q-RT) PCR. Results indicated that ATP7A, ATP7B and MDR1 were undetectable in A549 and A549/DDP cells (data not shown). The expression of GST- $\pi$ significantly increased and, whereas that of hCTR1significantly reduced in A549/DDP $(P<0.05)$. The expression of ERCC1 did not show significant difference between A549 and A549/DDP (Figure 1A). These results indicate that hCTR1 and GST- $\pi$ might participate in the DDP resistance of A549/DDP cells. The protein levels of GST- $\pi$ and hCTR1 were further examined by Western blot. The GST- $\pi$ expression in A549/DDP was higher compared with that in A549, whereas the CTR1 expression was lower (Figure 1B).

After co-culture with CIK cells for 6, 12, 16, and $20 \mathrm{~h}$, the gene expression levels of hCTR1 and GST- $\pi$ in A549/DDP were analyzed by q-RT-PCR. The relative gene expression of GST- $\pi$ in A549/DDP significantly decreased after co-culture with CIK cells $(P<0.05)$. The expression level of hCTR1 before and after co-culture with CIK cells exhibited no significant difference $(P>0.05)$ (Figure 2A). Furthermore, the proteins expression levels of GST- $\pi$ and hCTR1 in A549, A549/DDP, and A549/DDP co-cultured with CIK cells for $6,12,16$ and $20 \mathrm{~h}$ were examined by Western blot. After co-culture with CIK cells, GST- $\pi$ protein expression in A549/DDP decreased significantly $(P<0.05)$, which was consistent with the decreased RNA levels of GST- $\pi$. The hCTR1 expression in A549/DDP before and after co-culture with CIK cells showed no significant changes (Figure 2B).

\section{Effect of cytokines on reversal DDP resistance in A549/DDP co-cultured with CIK cells}

The secretion levels of TNF- $\alpha$, IL-2 and IFN- $\gamma$ in the supernatant of A549/DDP co-cultured with CIK cells were detected by ELISA. Results showed that the secretion of IFN- $\gamma$ significantly increased along with the co-culture time (Figure 3A). Correlation analysis showed that the DDP resistance reversal in A549/DDP correlated with the secretion level of IFN- $\gamma(\mathrm{r}=0.993, P=0.007)$, but not with the levels of TNF-a or IL-2 (data not shown).

To detect the effect of IFN- $\gamma$ on reversing DDP resistance, we detected the IC50 of DDP in A549, A549/DDP, and A549/DDP co-cultured with CIK cells in the absence or presence of anti-IFN- $\gamma$ neutralizing antibody. Results showed that the IC50 of A549/DDP co-cultured with CIK cells for 20h significantly increased after the addition of anti-IFN- $\gamma$ neutralizing antibody $(P<0.05)$ (Table 3$)$, indicating that CIK cells reversed the drug resistance of A549/DDP through the effects of IFN- $\gamma$. No significant difference was found between the 1 anti $5 \mu \mathrm{g} / \mathrm{ml}$ anti-IFN- $\gamma$ antibody groups (Table 3 ). The possible reason maybe that the lower concentration was sufficient to block CIK-secreted IFN- $\gamma$ completely.

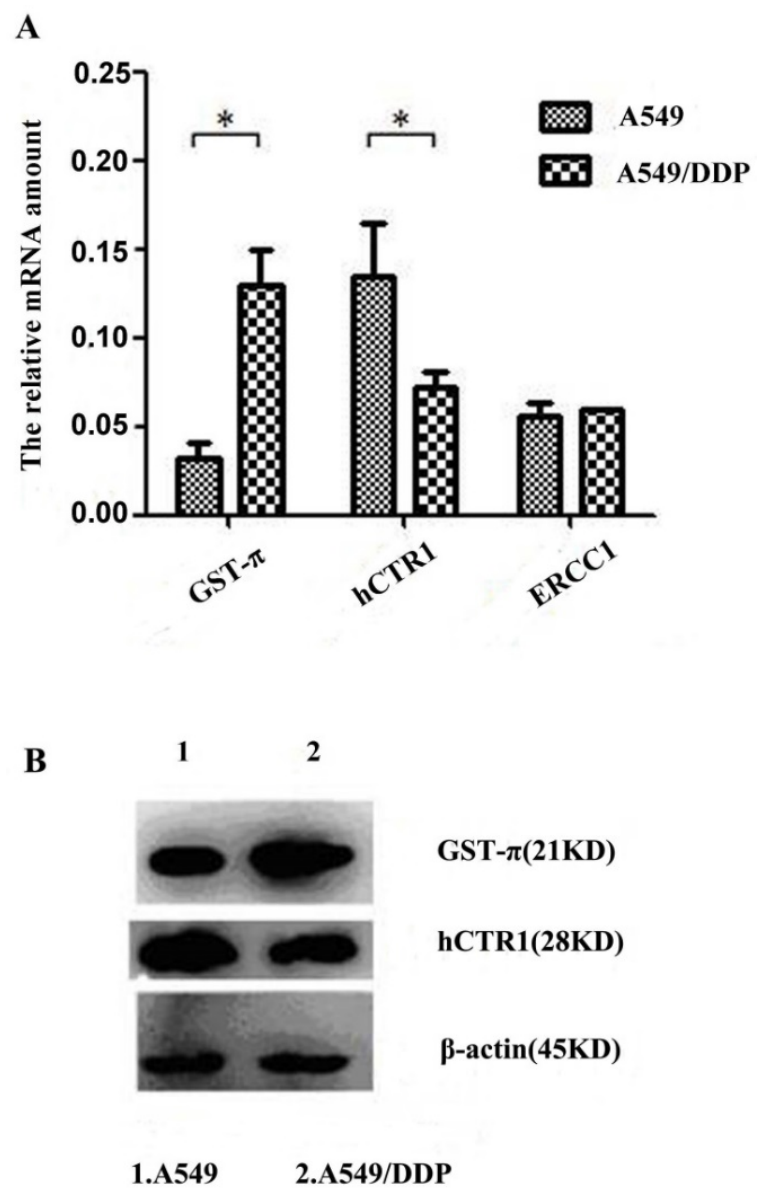

Figure 1. Analysis of drug resistance related genes of A549/DDP after co-culture with CIK cells A): Expression levels of hCTR1, GST- $\pi$ and ERCC1 in A549 and A549/DDP were analyzed by real-time PCR. Expression of GST- $\pi$ was significantly increased and hCTR1 expression was significantly reduced in A549/DDP $(P<0.05)$. The expression of ERCCl did not show significant difference between the two cell lines. B): Protein levels of GST- $\pi$ and hCTR1 were examined by Western Blot. GST- $\pi$ expression in A549/DDP was higher compared with that in A549 and hCTR1 expression was reduced. 


\section{A}

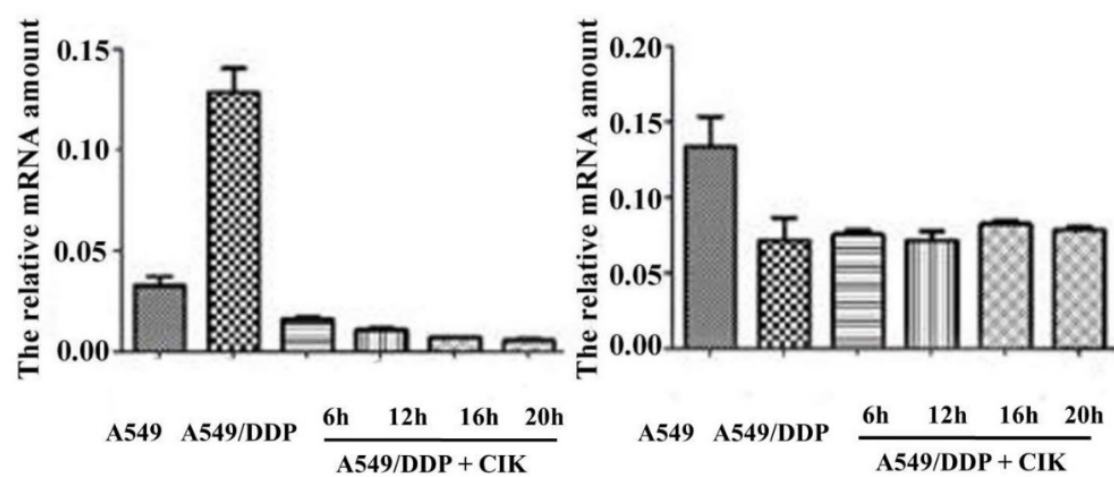

GST- $\pi$

hCTR1

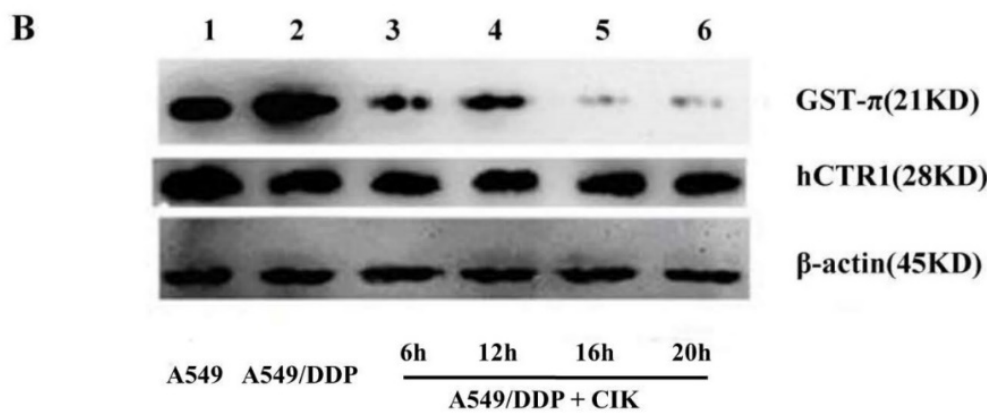

Figure 2. Alteration of the expression of GST- $\pi$ and hCTR1 in A549/DDP after co-culture with CIK cells A): Relative expression of GST- $\pi$ in A549/DDP significantly decreased after co-culture with CIK cells for $6,12,16$, and $20 \mathrm{~h}(P<0.05)$. The expression levels of $\mathrm{hCTR} 1$ before and after co-culture with CIK cells had no significant difference (P>0.05). B): The expression of GST- $\pi$ and hCTR1 proteins in A549, A549/DDP, and A549/DDP co-cultured with CIK cells for various times was examined by Western blot. After co-culture with CIK cells, the GST- $\pi$ expression in A549/DDP decreased.

Table 3. Effects of IFN- $\gamma$ neutralization on the re-sensitized drug response induced by $\mathrm{CIK}$

\begin{tabular}{lcll}
\hline & IC50 $(\mu \mathrm{M})$ & $\begin{array}{l}\text { Resistance } \\
\text { index }\end{array}$ & $\begin{array}{l}\text { Resistance } \\
\text { reversal index }\end{array}$ \\
\hline A549 & $5 \pm 1.25$ & & \\
A549/DDP & $74.5 \pm 5.24$ & 14.9 & \\
A549/DDP co-cultured for 20h & $15.6 \pm 2.89^{*}$ & 3.12 & 4.77 \\
$1 \mu \mathrm{g} / \mathrm{mL}$ anti-IFN- $\gamma$ antibody & $63.97 \pm 3.07$ & 12.79 & 1.16 \\
$5 \mu \mathrm{g} / \mathrm{mL}$ anti-IFN- $\gamma$ antibody & $60.26 \pm 4.42$ & 12.05 & 1.23 \\
\hline
\end{tabular}

Results are presented as mean \pm SD. We used one-way ANOVA for comparisons between different cell lines under different conditions. ${ }^{*} P<0.05$ compared with A549/DDP alone.

The GST- $\pi$ gene expression in the A549, A549/DDP, and A549/DDP cells co-cultured with CIK cells for $20 \mathrm{~h}$ with or without anti-IFN- $\gamma$ neutralizing antibody was examined by qRT- PCR. The expression of GST- $\pi$ was restored by addition of the neutralizing IFN- $\gamma$ (Figure 3B).

\section{DDP accumulation}

DDP accumulation was analyzed by HPLC. A symmetrical peak for typical chromatograms of DDP was shown, and the retention time for DDP was about 20.05 min (Figure 4A). A typical linear relationship $\left(\mathrm{R}^{2}=0.998\right)$ was found between the peak area and gradient concentration of DDP. The equation obtained from this calibration curve was $y=36690 x-8058.7$, where $y$ stands for the peak area of DDP and $x$ stands for the concentration of DDP. On the basis of this equation, the concentration of DDP in each cell line was determined. Results showed that the intracellular accumulation concentrations of DDP in A549 and A549/DDP were $1.5 \pm 0.03$ and $0.29 \pm 0.02 \mu \mathrm{g} / \mathrm{mL}$, respectively. After co-culture with CIK cells for $20 \mathrm{~h}$, the intracellular accumulation of DDP in A549/DDP significantly increased to $0.99 \pm 0.02 \mu \mathrm{g} / \mathrm{mL}$ compared with that in A549/DDP. After addition of the neutralizing IFN- $\gamma$ antibody, the intracellular accumulation of DDP decreased to $0.5 \pm 0.01 \mu \mathrm{g} / \mathrm{mL}$ (Figure 4B). 


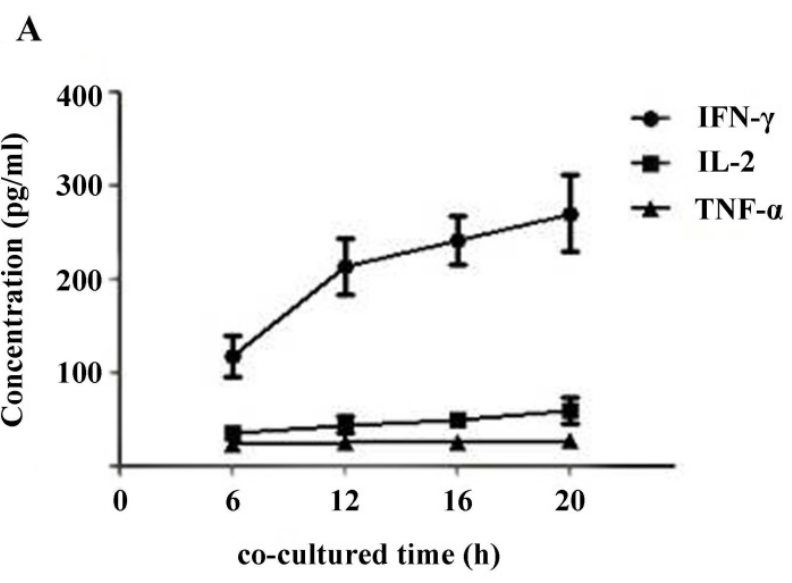

B

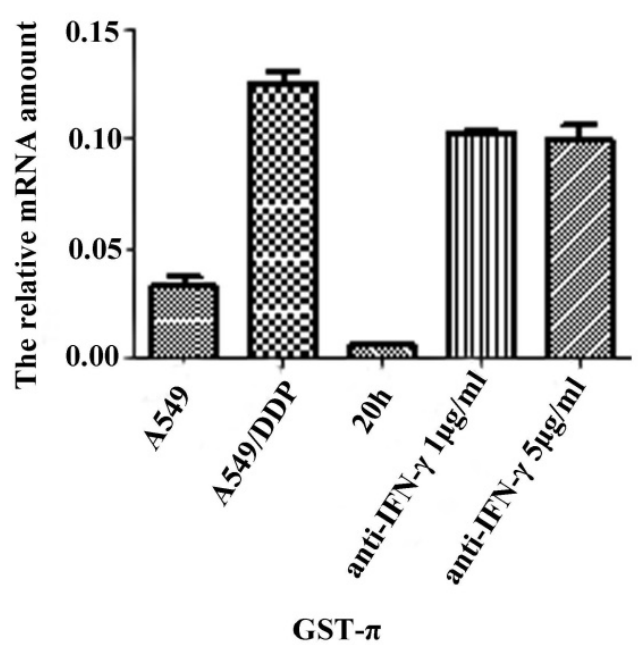

Figure 3. Effects of cytokine on reversal DDP resistance in A549/DDP co-cultured with CIK cells A): Cytokine secretion levels in CIK cells co-cultured with A549/DDP. The secretion levels of TNF- $\alpha$, IL-2 and IFN- $\gamma$ in the supernatant of A549/DDP co-cultured with CIK cells were detected by ELISA. The results showed that the secretion of IFN- $\gamma$ significantly increased along with the time of co-culture. B): Effects of IFN- $\gamma$ neutralization on GST- $\pi$ gene expression in A549/DDP. The GST- $\pi$ gene expression in A549, A549/DDP, and A549/DDP co-cultured with CIK cells for $20 \mathrm{~h}$ in the presence or absence of anti-IFN- $\gamma$ neutralizing antibody was examined by real-time PCR. GST- $\pi$ gene expression in A549/DDP co-cultured with CIK cells decreased compared with that in A549/DDP. However, neutralization of IFN- $\gamma$ significantly increased the expression of GST- $\pi$.

\section{Discussion}

Chemotherapy is the first-line cancer treatment, but its efficacy is hindered by the resistance of some tumor cells to chemotherapy drugs. Thus, the reversal of resistance to chemotherapy has become an important research area in recent years.

Drug resistance limits the application of DDP in NSCLC. Possible mechanisms of acquired resistance to DDP include reduced intracellular accumulation of DDP [11], enhanced drug inactivation by metallothionein and glutathione [12], increased activity of DNA damage repair [13] and altered expression of oncogenes and regulatory proteins. Several factors mediating the drug resistant phenotype are GST-ח, MDR1, hCTR1, ERCC1 and the copper transporters ATP7A and ATP7. Among the reported mechanisms that contribute to drug resistance, one of the most important is the overexpression of certain ATP-binding cassette (ABC) transporters in cancer cells leading to enhanced efflux of a large variety of therapeutic agents [14-16]. ABC transporters play a crucial role in drug absorption, distribution and excretion. Among the $A B C$ transporters, P-glycoprotein (Pgp, MDR1, ABCB1), multi-drug resistance protein 1 (MRP1, $\mathrm{ABCC} 1$ ) and breast cancer resistance protein (BCRP, ABCG2) are responsible for the majority of the observed efflux transporter-mediated drug resistance in human and animals $[17,18]$. In the present study, we only selected the MDR1gene of $\mathrm{ABC}$ transporters and found that MDR1 was undetectable. Previous studies have associated MDR1 overexpression with drug resistance in various tumor types [19, 20]. ERCC1 is a key enzyme in the nucleotide excision repair, which is essential for the removal of platinum-DNA adducts. Meanwhile, clinical studies have associated the increased expression of ERCC1 with resistance to platinum-based chemotherapy and worse prognosis in patients with advanced NSCLC [21, 22] and in patients with completely resected NSCLC [23]. hCTR1, ATP7A and ATP7B are associated with the transportation of copper ions. Recent studies have shown that hCTR1, ATP7A and ATP7B are involved in platinum drug transportation; alterations in the expression patterns of these proteins are related to DDP resistance in tumors [24, 25]. Targeting different mechanisms of drug resistance, various agents that can reverse chemotherapy resistance have been identified [26-28]. Some studies suggested that CIK cells play a role in reversing the drug resistance in chemoresistant cells. Zhao et al. [7] found that the killing activity of CIK cells combined with oxaliplatin in oxaliplatin-resistant gastric cancer cells OCUM-2MD3/L-OHP is significantly higher than those of the two single-agent treatments. In vivo studies showed that the survival rate of mice that received combined treatment is significantly higher than those of mice that received separate treatments. Liu et al. [6] reported that CIK cells in combination with docetaxel significantly sensitize the docetaxel-resistant cell line SPC-A1/DTX to the treatment both in vitro and in vivo.

The present study indicated that the sensitivity of A549/DDP to DDP significantly increased after co-culture with CIK cells. The GST- $\pi$ expression in A549/DDP co-cultured with CIK cells significantly decreased at the mRNA and protein levels. The 
expression of hCTR1 did not show any change. GST- $\pi$ has been involved in DDP resistance; several studies have shown that GST- $\pi$ is highly expressed in drug resistant tumor cells [29-32]. GST- $\pi$, a protein involved in transfer and cell metabolism of toxins, including chemotherapy drugs, can cause drug resistance by promoting drug efflux in tumor cells [12]. hCTR1 is the main influx transporter of copper ions. Recent studies have shown that hCTR1 is also involved in platinum drug intake. Decreased hCTR1 expression has been observed in DDP-resistant cells, and has been associated with DDP resistance [25]. The present results showed that CIK cells reversed the DDP resistance through GST- $\pi$ and not hCTR1.

In the present study, the potential mechanisms underlying the drug re-sensitizing effect of CIK cells were examined. We focused on the cytokines secreted by CIK cells. Correlation analysis demonstrated that the IFN- $\gamma$ secreted by CIK cells and the reversal of DDP resistance in A549/DDP were statistically correlated, whereas no correlation was detected between the levels of TNF- $\alpha$ or IL-2 and the drug response. When IFN- $\gamma$ was neutralized by an antibody, the resistance reversal effects of CIK cells were significantly decreased, and the repressed expression of GST- $\pi$ in A549/DDP was elevated. The effects of CIK co-culture on re-sensitizing A549/DDP to DDP treatment and the GST- $\pi$ expression in the cells were not affected by neutralizing antibodies against TNF- $\alpha$ or IL-2. These results suggest that CIK cells reverse the DDP resistance in A549/DDP by reducing GST- $\pi$ expression through a mechanism mediated by CIK-secreted IFN- $\gamma$.

Many studies have linked cytokines to reversed chemoresistance in tumor cells. Wadler et al. [33] combined chemotherapy drugs with cytokines to treat drug-resistant cells in vitro, and found that cytokines can change the resistance phenotype of cancer cells to restore chemosensitivity. Liu et al. [34] proposed that IFN-a can reduce MDR1 and MRP expression in hepatocarcinoma cells, and thus reverse the drug resistance. Manara et al. [35] also showed that IFN-a can down-regulate MDR1 expression in ADM-resistant osteosarcoma cells to increase the cell sensitivity to ADM. In addition, Cao et al. [36] have combined TNF-a with doxorubicin in the treatment of multidrug resistant MCF-7/Adr cell line, and found that TNF-a promotes doxorubicin-induced cell death and anti-cancer effect. Li et al. [37] reported that TNF-a possibly inhibits the expression of XIAP, survivin, PCNA, BCRP and other genes related to drug resistance to achieve re-sensitized drug

A
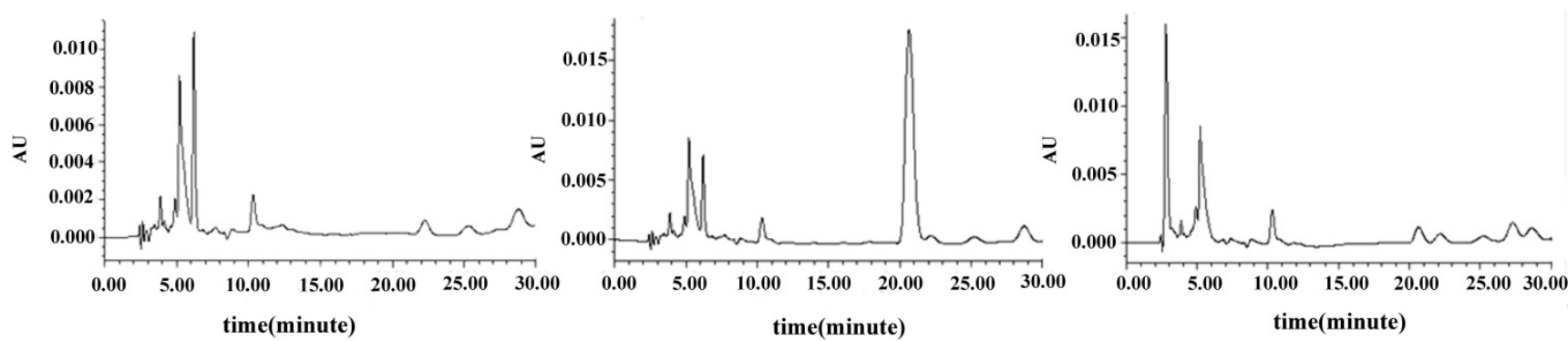

B

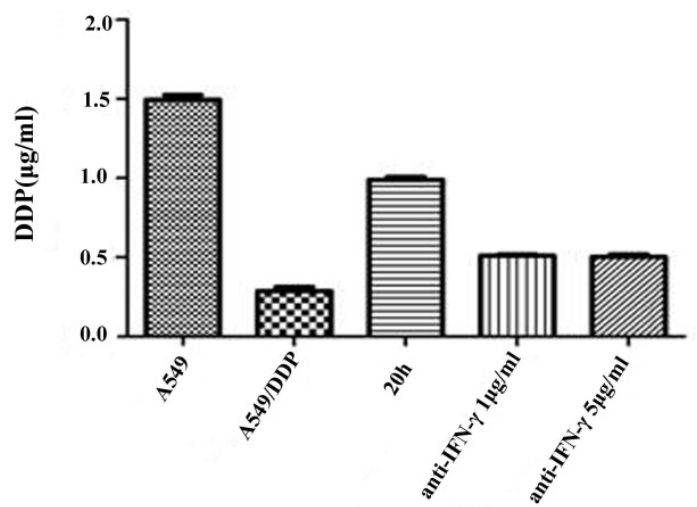

Figure 4. Chromatograms of DDP in different groups A): DDP accumulation was analyzed by HPLC. A symmetrical peak for typical chromatograms of DDP was shown, and the retention time for the DDP was about $20.05 \mathrm{~min}$. B): After co-culture with CIK cells for $20 \mathrm{~h}$, the intracellular accumulation of DDP in A549/DDP significantly increased compared with that in A549/DDP. After addition of the neutralizing IFN- $\gamma$ antibody, the intracellular accumulation of DDP decreased. 
response. Our experiments demonstrated the significant correlation between IFN- $\gamma$ and DDP re-sensitization in A549/DDP.

In summary, our study showed that CIK cells reversed the DDP resistance and reduced the expression of GST- $\pi$ in A549/DDP which is dependent on IFN- $\gamma$ secreted by CIK cells. IFN- $\gamma$ reduced GST- $\pi$ expression in A549/DDP to decrease the intracellular concentration of DDP and reverse DDP resistance. However, the mechanism by which IFN- $\gamma$ regulates GST- $\pi$ expression remains unclear. Further experiments are required to decipher this IFN- $\gamma$-mediated mechanism. In our future studies, we will use cell lines from other cancer types and surgically resect human tumor samples to determine the reliability and the specificity of the findings.

\section{Abbreviations}

CIKs: cytokine-induced killer cells; DDP: cisplatin; RI: resistance index; NSCLC: Non small cell lung cancer; PBMCs: peripheral blood mononuclear cells; IR: inhibitory rate; GST-п: glutathione-S-transferase ח; MDR1: multidrug resistance 1; hCTR1: human copper transporter 1; ERCC1: excision repair cross complementing 1; HPLC: high-performance liquid chromatography; q-RT: quantitative real time; ABC: ATP-binding cassette; NER: nucleotide excision repair.

\section{Acknowledgements}

This work was supported by a grant from National Key Technology R\&D Program (No.2015BAI12B12), key projects of Tianjin health industry (No.15KG145) and national natural science foundation of china (81572265 \& 31500736).

\section{Competing Interests}

The authors have declared that no competing interest exists.

\section{References}

1. Wood SL, Pernemalm M, Crosbie PA, Whetton AD. Molecular histology of lung cancer: from targets to treatments. Cancer treatment reviews. 2015; 41: 361-75.

2. Reck M, Popat S, Reinmuth N, De Ruysscher D, Kerr KM, Peters S. Metastatic non-small-cell lung cancer (NSCLC): ESMO Clinical Practice Guidelines for diagnosis, treatment and follow-up. Annals of oncology: official journal of the European Society for Medical Oncology / ESMO. 2014; 25 Suppl 3: iii27-39.

3. Wang G, Reed E, Li QQ. Molecular basis of cellular response to cisplatin chemotherapy in non-small cell lung cancer (Review). Oncology reports. 2004; 12: 955-65.

4. Mesiano G, Todorovic M, Gammaitoni L, Leuci V, Giraudo Diego L, Carnevale-Schianca F, et al. Cytokine-induced killer (CIK) cells as feasible and effective adoptive immunotherapy for the treatment of solid tumors. Expert Opin Biol Ther. 2012; 12: 673-84.

5. Jakel CE, Schmidt-Wolf IG. An update on new adoptive immunotherapy strategies for solid tumors with cytokine-induced killer cells. Expert Opin Biol Ther. 2014; 14: 905-16.

6. Liu $\mathrm{P}$, Chen $\mathrm{L}$, Huang $X$. The antitumor effects of CIK cells combined with docetaxel against drug-resistant lung adenocarcinoma cell line SPC-A1/DTX in vitro and in vivo. Cancer biotherapy \& radiopharmaceuticals. 2009; 24: 91-8.
7. Zhao Q, Zhang H, Li Y, Liu J, Hu X, Fan L. Anti-tumor effects of CIK combined with oxaliplatin in human oxaliplatin-resistant gastric cancer cells in vivo and in vitro. Journal of experimental \& clinical cancer research: CR. 2010; 29: 118.

8. Yang L, Ren B, Li H, Yu J, Cao S, Hao X, et al. Enhanced antitumor effects of DC-activated CIKs to chemotherapy treatment in a single cohort of advanced non-small-cell lung cancer patients. Cancer immunology, immunotherapy: CII. 2013; 62: 65-73.

9. Ren X, Yu J, Liu H, Zhang P, An X, Zhang N, et al. Th1 bias in PBMC induced by multicycles of auto-CIKs infusion in malignant solid tumor patients. Cancer biotherapy \& radiopharmaceuticals. 2006; 21: 22-33.

10. Schmidt-Wolf IG, Finke S, Trojaneck B, Denkena A, Lefterova P, Schwella N, et al. Phase I clinical study applying autologous immunological effector cells transfected with the interleukin-2 gene in patients with metastatic renal cancer, colorectal cancer and lymphoma. British journal of cancer. 1999; 81: 1009-16.

11. Pham AN, Wang J, Fang J, Gao X, Zhang $Y$, Blower PE, et al. Pharmacogenomics approach reveals MRP1 (ABCC1)-mediated resistance to geldanamycins. Pharmaceutical research. 2009; 26: 936-45.

12. Federici L, Lo Sterzo C, Pezzola S, Di Matteo A, Scaloni F, Federici G, et al. Structural basis for the binding of the anticancer compound 6-(7-nitro-2,1,3-benzoxadiazol-4-ylthio)hexanol to human glutathione s-transferases. Cancer research. 2009; 69: 8025-34.

13. Chiu TJ, Chen CH, Chien CY, Li SH, Tsai HT, Chen YJ. High ERCC1 expression predicts cisplatin-based chemotherapy resistance and poor outcome in unresectable squamous cell carcinoma of head and neck in a betel-chewing area. Journal of translational medicine. 2011; 9: 31.

14. Konig J, Muller F, Fromm MF. Transporters and drug-drug interactions: important determinants of drug disposition and effects. Pharmacological reviews. 2013; 65: 944-66.

15. Deng J, Shao J, Markowitz JS, An G. ABC transporters in multi-drug resistance and ADME-Tox of small molecule tyrosine kinase inhibitors. Pharmaceutical research. 2014; 31: 2237-55.

16. He M, Wei MJ. Reversing multidrug resistance by tyrosine kinase inhibitors. Chinese journal of cancer. 2012; 31: 126-33.

17. Szakacs G, Varadi A, Ozvegy-Laczka C, Sarkadi B. The role of ABC transporters in drug absorption, distribution, metabolism, excretion and toxicity (ADME-Tox). Drug discovery today. 2008; 13: 379-93.

18. Lagas JS, van Waterschoot RA, van Tilburg VA, Hillebrand MJ, Lankheet N, Rosing $\mathrm{H}$, et al. Brain accumulation of dasatinib is restricted by P-glycoprotein (ABCB1) and breast cancer resistance protein (ABCG2) and can be enhanced by elacridar treatment. Clinical cancer research: an official journal of the American Association for Cancer Research. 2009; 15: 2344-51.

19. Sanchez C, Mercado A, Contreras HR, Mendoza P, Cabezas J, Acevedo C, et al. Chemotherapy sensitivity recovery of prostate cancer cells by functional inhibition and knock down of multidrug resistance proteins. The Prostate. 2011; 71: 1810-7

20. Angelini A, Centurione L, Sancilio S, Castellani ML, Conti P, Di Ilio C, et al. The effect of the plasticizer diethylhexyl phthalate on transport activity and expression of P-glycoprotein in parental and doxo-resistant human sarcoma cell lines. Journal of biological regulators and homeostatic agents. 2011; 25: 203-11.

21. Ceppi P, Volante M, Novello S, Rapa I, Danenberg KD, Danenberg PV, et al. ERCC1 and RRM1 gene expressions but not EGFR are predictive of shorter survival in advanced non-small-cell lung cancer treated with cisplatin and gemcitabine. Annals of oncology: official journal of the European Society for Medical Oncology / ESMO. 2006; 17: 1818-25.

22. Lord RV, Brabender J, Gandara D, Alberola V, Camps C, Domine M, et al. Low ERCC1 expression correlates with prolonged survival after cisplatin plus gemcitabine chemotherapy in non-small cell lung cancer. Clinical cancer research: an official journal of the American Association for Cancer Research. 2002; 8: 2286-91.

23. Olaussen KA, Dunant A, Fouret P, Brambilla E, Andre F, Haddad V, et al. DNA repair by ERCC1 in non-small-cell lung cancer and cisplatin-based adjuvant chemotherapy. The New England journal of medicine. 2006; 355: 983-91.

24. Rabik CA, Maryon EB, Kasza K, Shafer JT, Bartnik CM, Dolan ME. Role of copper transporters in resistance to platinating agents. Cancer chemotherapy and pharmacology. 2009; 64: 133-42.

25. Song IS, Savaraj N, Siddik ZH, Liu P, Wei Y, Wu CI, et al. Role of human copper transporter Ctr1 in the transport of platinum-based antitumor agents in cisplatin-sensitive and cisplatin-resistant cells. Molecular cancer therapeutics. 2004; 3: 1543-9.

26. Johansson $\mathrm{K}$, Ito $\mathrm{M}$, Schophuizen $\mathrm{CM}$, Mathew Thengumtharayil S, Heuser $\mathrm{VD}$, Zhang J, et al. Characterization of new potential anticancer drugs designed to overcome glutathione transferase mediated resistance. Molecular pharmaceutics. 2011; 8: 1698-708.

27. Angelini A, Iezzi M, Di Febbo C, Di Ilio C, Cuccurullo F, Porreca E. Reversal of P-glycoprotein-mediated multidrug resistance in human sarcoma MES-SA/Dx-5 cells by nonsteroidal anti-inflammatory drugs. Oncology reports. 2008; 20: 731-5.

28. Donmez Y, Akhmetova L, Iseri OD, Kars MD, Gunduz U. Effect of MDR modulators verapamil and promethazine on gene expression levels of MDR1 and MRP1 in doxorubicin-resistant MCF-7 cells. Cancer chemotherapy and pharmacology. 2011; 67: 823-8. 
29. Wang J, Zhang J, Zhang L, Zhao L, Fan S, Yang Z, et al. Expression of P-gp, MRP, LRP, GST-pi and TopoIIalpha and intrinsic resistance in human lung cancer cell lines. Oncol Rep. 2011; 26: 1081-9.

30. Geng $\mathrm{M}$, Wang $\mathrm{L}$, Chen $\mathrm{X}$, Cao $\mathrm{R}$, Li P. The association between chemosensitivity and Pgp, GST-pi and Topo II expression in gastric cancer. Diagnostic pathology. 2013; 8: 198.

31. Patel N, Chatterjee SK, Vrbanac V, Chung I, Mu CJ, Olsen RR, et al. Rescue of paclitaxel sensitivity by repression of Prohibitin1 in drug-resistant cancer cells. Proceedings of the National Academy of Sciences of the United States of America. 2010; 107: 2503-8.

32. Cullen KJ, Newkirk KA, Schumaker LM, Aldosari N, Rone JD, Haddad BR. Glutathione S-transferase pi amplification is associated with cisplatin resistance in head and neck squamous cell carcinoma cell lines and primary tumors. Cancer research. 2003; 63: 8097-102

33. Takeuchi A, Kaneko S, Matsushita E, Urabe T, Shimoda A, Kobayashi K. Interferon-alpha modulates resistance to cisplatin in three human hepatoma cell lines. Journal of gastroenterology. 1999; 34: 351-8.

34. Liu Y, Lou G, Wu W, Shi $Y$, Zheng M, Chen Z. Interferon-alpha sensitizes HBx-expressing hepatocarcinoma cells to chemotherapeutic drugs through inhibition of HBx-mediated NF-kappaB activation. Virology journal. 2013; 10: 168.

35. Manara MC, Serra M, Benini S, Picci P, Scotlandi K. Effectiveness of Type I interferons in the treatment of multidrug resistant osteosarcoma cells. International journal of oncology. 2004; 24: 365-72.

36. Cao W, Ma SL, Tang J, Shi J, Lu Y. A combined treatment TNF-alpha/doxorubicin alleviates the resistance of MCF-7/Adr cells to cytotoxic treatment. Biochimica et biophysica acta. 2006; 1763: 182-7.

37. Li G, Dong S, Qu J, Sun Z, Huang Z, Ye L, et al. Synergism of hydroxyapatite nanoparticles and recombinant mutant human tumour necrosis factor-alpha in chemotherapy of multidrug-resistant hepatocellular carcinoma. Liver international: official journal of the International Association for the Study of the Liver. 2010; 30: 585-92. 